\section{Efeito dos treinamentos aeróbio, resistido e concorrente na pressão arterial e morfologia de idosos normotensos e hipertensos}

\author{
Effect of aerobic, resistance and concurrent \\ training on blood pressure and morphology \\ of normotensive and hypertensive elderly \\ people
}

Paulo Roberto Cavalcanti Carvalho ${ }^{1}$

Gustavo Willames Pimentel Barros ${ }^{1}$

Thamara Thais Santos de Melo ${ }^{1}$

Petrus Gantois Massa Dias dos Santos ${ }^{1}$

Gledson Tavares de Amorim Oliveira ${ }^{1}$

Igor de Rodrigues D'Amorim ${ }^{1}$

\begin{abstract}
Resumo
Introdução: A Hipertensão Arterial (HA) é considerada um dos fatores de risco mais ostensivos para o desenvolvimento de doenças cardiovasculares. Representando no Brasil um dos problemas de saúde pública de maior prevalência na população, capaz de levar a óbito aproximadamente 40\% dos indivíduos acometidos. Diante do exposto, deve-se dar uma ênfase cada vez maior à prática de exercícios físicos como fator determinante na redução da pressão arterial, resultando em Hipotensão Pós-Exercício (HPE). Objetivo: Verificar o efeito dos treinamentos aeróbio, resistido e concorrente na pressão arterial e morfologia em idosos normotensos e hipertensos. Métodos: A amostra foi composta por 300 idosos, normotensos $(n=150)$ e hipertensos $(n=150)$, divididos em três grupos. O Grupo 1 (G1) realizou treinamento aeróbio; o Grupo 2 (G2) treinamento resistido e o Grupo 3 (G3) treinamento concorrente. Para comparar as variáveis de cada grupo no período pré e pós-treinamento foi utilizado o teste $t$ para amostras pareadas. $\mathrm{O}$ nível de significância estabelecido foi de $\mathrm{p}<0,05$. Resultados: Foi observada redução da pressão arterial pós-exercício em todos os grupos, apenas para a pressão arterial sistólica, tendo efeito hipotensor para os normotensos do G1, enquanto os hipertensos obtiveram redução da pressão arterial sistólica para os três grupos. Para a morfologia não foram verificadas diferenças significativas. Conclusão: Após o término das sessões de treinamento ficou evidenciada a importância da prática regular de exercício físico, seja ele aeróbio, resistido ou concorrente, na prevenção e no combate da HA.
\end{abstract}

\section{Palavras-chave}

Saúde; Antropometria; Exercício; Envelhecimento; Treinamento.

\begin{abstract}
Introduction: Hypertension $(H A)$ is considered one of the risk factors for the development of overt cardiovascular disease. Representing in Brazil one of the major problems of public health with the biggest prevalence in population, capable of leading to death $40 \%$ of the affected individuals. Therefore, we must take a growing emphasis on physical exercise as a factor in reducing BP, resulting in post-exercise hypotension (PEH). Objective: To verify the effect of aerobic, resistance and concurrent training on blood pressure and morphology in normotensive and hypertensive elderly people. Methods: The sample was composed by 300 elderly people, normotensive $(n=150)$ and bypertensive $(n=150)$, divided into three groups. Group 1 (G1) performed aerobic training, Group 2 (G2) resistance training and Group 3 (G3) concurrent training. To compare the variable in each group in the pre and post-training periods, it was used $t$-test for paired samples. The significance level established was $p<0.05$. Results: It was observed reduction of the blood pressure post-exercise in all the groups, only for systolic blood pressure, causing bypotensive effect for the normotensive group (G1), while the hypertensive people had reduction of systolic blood pressure in the three groups. For morphology not significant differences was verified. Conclusion: After the end of training sessions was evident the importance of regular practice of physical exercise, whether it be aerobic, resistance or concurrent in preventing and combating the arterial hypertension.
\end{abstract}

\section{Keywords}

Health; Anthropometry; Exercise; Aging; Training.
Rev Bras Ativ Fis Saúde p. 363-370 DOI:

http://dx.doi.org/10.12820/rbafs.v.18n3p363

1 Departamento de Educação Física. Laboratório de Atividade Física e Saúde, Universidade Federal de Pernambuco. 


\section{INTRODUÇÃO}

A Hipertensão Arterial (HA) é considerada um dos fatores de risco mais ostensivos para o desenvolvimento de doenças cardiovasculares ${ }^{1,2}$, sendo considerada uma síndrome multifatorial que afeta mais de um quarto da população mundial, podendo acometer aproximadamente $29 \%$ destes indivíduos até $2025^{3}$. No Brasil, no ano de 2006 , a HA atingiu cerca de $22 \%$ da população adulta ${ }^{4}$, enquanto que analisado entre a população idosa, este número pode variar de 50 a $70 \%$ de indivíduos acometidos ${ }^{5}$.

Alguns estudos têm demonstrado associação entre a HA e indicadores antropométricos relacionados ao excesso de gordura corporal, localizada principalmente na região abdominal ${ }^{6,7}$ como confirma Neter et al. ${ }^{8}$, em uma meta-análise constituída por 25 estudos clínicos randomizados, no qual identificaram uma redução de aproximadamente 1,1 $\mathrm{mmHg}$ na pressão arterial sistólica (PAS) e de 0,9 $\mathrm{mmHg}$ na pressão arterial diastólica (PAD), para cada $1 \mathrm{~kg}$ de peso reduzido. Dessa forma, o monitoramento e o controle do peso corporal torna-se uma medida eficaz para a prevenção e tratamento de doenças crônico-degenerativas como a HA 9 .

Diante do exposto, ênfase cada vez maior vem sendo dada à prática de exercícios físicos como fator determinante na redução da pressão arterial $(\mathrm{PA})^{10}$, resultando em HPE. A eficácia da HPE no exercício aeróbio, tanto para indivíduos normotensos, sendo utilizado de forma preventiva ${ }^{11}$, como para hipertensos, como um tratamento não farmacológico para a HA, já é um fato bastante evidenciado na literatura ${ }^{2,12-14}$.

No entanto, quando se trata de exercícios resistidos, os resultados para HPE ainda são contraditórios ${ }^{11,15}$. Isso ocorre porque durante muito tempo acreditava-se que o efeito do treinamento resistido pudesse causar uma elevação da PA decorrente de uma provável hipertrofia do músculo liso vascular ${ }^{16}$. Contudo, nos últimos anos, estudos têm demonstrado redução da PA em sujeitos hipertensos e normotensos submetidos a este tipo de treinamento ${ }^{17,18}$, como em uma meta-análise realizada por Kelley; Kelley ${ }^{19}$ que evidenciaram a eficácia do treinamento resistido na redução da $\mathrm{PA}$.

Desta forma, o objetivo do presente estudo foi verificar o efeito do treinamento aeróbio, resistido e concorrente (aeróbio + resistido) sobre a PA e a morfologia de idosos normotensos e hipertensos.

\section{METODOLOGIA}

\section{Tipo de estudo e amostra}

A presente pesquisa é um estudo do tipo transversal com amostragem por conveniência, realizada no Departamento de Educação Física da Universidade Federal de Pernambuco no Recife/PE, sendo que os indivíduos estudados faziam parte do projeto QualisVida.

A amostra foi composta por 300 idosos, sendo hipertensos ( $n=150)$ e normotensos $(n=150)$, todos sem impedimentos físicos. Os idosos que participaram do estudo assinaram um Termo de Consentimento Livre e Esclarecido (TCLE), de acordo com as normas estabelecidas pela resolução 196/96 do Conselho Nacional de Saúde para pesquisas envolvendo seres humanos, com critérios de inclusão considerados indivíduos que não possuíam qualquer outra enfermidade que pudesse comprometer as respostas cardiovasculares e/ou limitações articulares que impossibilitassem a realização dos exercícios. $\mathrm{O}$ estudo foi aprovado pelo Comitê de Ética do Centro de Ciências da Saúde, da Universidade Federal de Pernambuco (processo 321/11). 


\section{Procedimento experimental}

A amostra foi dividida em três grupos de forma aleatória estratificada (normotenso/hipertenso), a fim de agregar 100 sujeitos em cada estrato por grupo. No que concerne à amostra inicial, apenas 267 idosos concluíram o protocolo de treinamento, havendo desta forma uma perda amostral de 33 participantes (11\%), por ausência de adaptabilidade aos treinamentos, inviabilidade de locomoção ao local do estudo ou por motivos pessoais. Desta forma o G1 foi composto por quarenta normotensos e quarenta e sete hipertensos, o G2 por quarenta e oito normotensos e quarenta e cinco hipertensos, enquanto o G3 ficou constituído por quarenta cinco normotensos e quarenta e dois hipertensos.

Cada grupo foi submetido a 36 sessões de treinamento, tendo o Grupo 1 (G1), realizado treinamento aeróbio, Grupo 2 (G2), treinamento resistido e Grupo 3 (G3), treinamento concorrente (aeróbio + resistido). A coleta de dados teve início em Janeiro de 2012 e término em maio de 2012.

\section{Avaliações antropométricas}

Para a identificação da composição corporal da amostra foi mensurado a massa corporal (kg) em uma balança portátil (Filizola) com resolução próxima de 0,1 kg, a estatura corporal através de uma estadiômetro (Sanny), com resolução próxima de $0,1 \mathrm{~cm}$, a fim de se obter o Índice de Massa Corporal IMC, bem como as circunferências da cintura (CC), do quadril (CQ) e do abdômen (CA), através de uma fita antropométrica não flexível (Sanny) para a estimação da relação cintura/ quadril (RCQ) e do índice de conicidade (IC). Todas as mensurações foram realizadas no período que antecedeu o início do treinamento e ao final das 36 sessões, seguindo as normas da ISAK ${ }^{21}$. As avaliações foram realizadas pelo mesmo avaliador com erro técnico de medida inferior a 5\% e coeficiente de teste-reteste acima de $r=0,95$ para todos os pontos anatômicos.

\section{Medida da Pressão Arterial}

A monitorização da pressão arterial no período pré e pós-treinamento foi realizada através dos métodos palpatório e auscultatório, com a utilização de um esfigmomanômetro analógico (BD e estetoscópio (BD» tipo MDF 747-duo sonic). A Pressão Arterial Sistólica (PAS) foi determinada de acordo com o $1^{\circ}$ ruído de korotkoff, e a Pressão Arterial Diastólica (PAD) correspondendo ao $5^{\circ}$ ruído. O padrão de medida da pressão arterial seguiu as recomendações da American Heart Association ${ }^{22}$. Antes das medidas pré-treino e pós-treino, os sujeitos foram submetidos a 5 minutos de descanso em posição sentada para o retorno ao estado basal. Os participantes foram orientados a ficar em silêncio no momento da aferição e a não ingerir bebidas alcoólicas e/ou cafeína num período inferior a 24 horas precedente a medida da PA. Todas as aferições foram realizadas por um único avaliador previamente treinado.

\section{Protocolo de treinamento:}

Primeiramente, todos os sujeitos realizaram duas semanas de adaptação para esclarecimentos sobre a correta execução dos exercícios, bem como a amplitude a ser realizada. Após o período de adaptação, todos os voluntários compareceram ao local do estudo para realizar o teste de $10 \mathrm{RM}^{23}$, a fim de obter uma estimativa da repetição máxima (1RM) de cada exercício; para tal, cada voluntário realizou no máximo 5 tentativas em cada exercício, com intervalo de 5 minutos entre cada uma ${ }^{24}$. Foi utilizada a escala de Borg para questionar sobre o esforço percebido com a intensidade da carga em cada tentativa, mantendo-se em fraca a moderada ${ }^{25}$. Cada sujeito foi 
submetido a 36 sessões de treinamento, sendo realizados três dias na semana, alternadamente. A cada grupo foi designado um tipo de treinamento com intensidade de leve a moderada, realizado entre $40 \%$ a $60 \%$ da frequência cardíaca máxima, com duração de 45 a 60 minutos. O G1 realizou treinamento aeróbio, sendo predominantemente caminhada, no qual os sujeitos caminhavam $3.200 \mathrm{~m}$ na pista de atletismo do Departamento de Educação Física da Universidade Federal de Pernambuco. O G2 realizou treinamento de força, do tipo alternado por segmento dividido em treino 'A' e 'B'; cada treino conteve seis exercícios. O treino 'A' foi composto pelos seguintes exercícios: supino vertical, leg press horizontal, tríceps no pulley, cadeira extensora, desenvolvimento e cadeira abdutora; já o treino 'B' foi composto por: puxada frontal, cadeira flexora, rosca bíceps direta, panturrilha no leg press, rosca punho supinada e cadeira adutora. O G3 realizou um treinamento concorrente, sendo no período da manhã realizado o treinamento aeróbio e à tarde, o treinamento resistido. No treinamento resistido os sujeitos realizaram três séries de 12 repetições, com intervalo de 45 segundos entre cada série, e 1 minuto entre cada exercício. Todos os treinamentos foram realizados no período da manhã, com exceção do G3, que foi exposto a dois treinos com a mesma duração dos outros grupos, realizada em dois turnos diferentes com um intervalo de $9 \mathrm{~h}$, sendo todos supervisionados por um professor e três estudantes de Educação Física, previamente treinados.

\section{Análise estatística:}

Para a verificação da normalidade das variáveis do estudo, foi utilizado o teste Kolmogorov-Smirnov Z. Os dados contínuos foram expressos em média e desvio padrão. A análise estatística foi realizada através do software Statistical Package for the Social Sciences - SPSS versão 17.0. O teste $t$ para amostras pareadas foi utilizado para a comparação das variáveis de cada grupo no período pré e pós-treinamento. O nível de significância estabelecido foi de $\mathrm{p}<0,05$.

\section{RESULTADOS}

No teste de Kolmogorov-Smirnov Z, apresentou resultados de normalidade para todas as variáveis estudadas.

Todos os sujeitos hipertensos faziam uso de medicamentos anti-hipertensivos, sendo estes diuréticos, tiazídicos, bloqueadores de canal de cálcio (diidropiridinas) e antagonistas do receptor angiotensina II, mas não houve homogeneidade quanto ao controle do fármaco.

A tabela 1 apresenta a caracterização da amostra em média, desvio padrão e frequência, de acordo com dados sociodemográficos, antropométricos e a média da PAS e PAD. Ao analisar as características sociodemográficas destaca-se a predominância das mulheres $(\mathrm{n}=25)$, com uma idade média de $(66,65 \pm 4,09)$ para a amostra total.

Os resultados da pressão arterial pré e pós-treino em sujeitos normotensos após 36 sessões de treinamento aeróbio (G1), resistido (G2) e concorrente (G3) está apresentado na tabela 2 . Ao verificar o efeito do treinamento na pressão arterial foi visto apenas efeito hipotensivo significativamente nos sujeitos submetidos ao treinamento aeróbio ( $\mathrm{p}=0,001)$ para a $\mathrm{PAS}$, com redução de $3,8 \mathrm{mmHg}$.

O efeito das 36 sessões de treinamento aeróbio (G1), resistido (G2) e concorrente (G3) na pressão arterial de sujeitos hipertensos pré e pós-treino está ilustrado na tabela 3. O efeito hipotensivo após o período de treinamento apresentou resultados significativos para os três grupos na PAS (G1, p=0,014; G2, p=0,004; $\mathrm{G} 3, \mathrm{p}=0,005)$, tendo o $\mathrm{G} 1$ apresentado a maior redução na PAS $(7,5 \mathrm{mmHg})$. 
Tabela 1 - Características gerais dos idosos submetidos a 36 sessões de treinamento aeróbio, resistido e concorrente.

\begin{tabular}{lccc}
\hline \multicolumn{1}{c}{ Variáveis } & G1 & G2 & G3 \\
\hline Idade & $68,14 \pm 5,03$ & $65,33 \pm 3,35$ & $65,66 \pm 2,34$ \\
\hline Homem & $10(11,5 \%)$ & $11(11,8 \%)$ & $09(10,4 \%)$ \\
\hline Mulher & $77(88,5 \%)$ & $82(88,2 \%)$ & $78(89,6 \%)$ \\
\hline Massa Corporal $(\mathrm{kg})$ & $64,15 \pm 13,22$ & $69,82 \pm 16,79$ & $79,84 \pm 14,39$ \\
\hline Estatura $(\mathrm{m})$ & $1,54 \pm 0,07$ & $1,57 \pm 0,13$ & $1,57 \pm 0,09$ \\
\hline IMC $\left(\mathrm{kg} / \mathrm{m}^{2}\right)$ & $26,68 \pm 4,34$ & $28,05 \pm 4,42$ & $32,23 \pm 4,46$ \\
\hline RCQ & $0,85 \pm 0,97$ & $0,92 \pm 0,15$ & $0,90 \pm 0,04$ \\
\hline IC & $1,25 \pm 0,22$ & $1,35 \pm 0,19$ & $1,47 \pm 0,15$ \\
\hline CC $(\mathrm{cm})$ & $83,56 \pm 13,39$ & $89,95 \pm 11,82$ & $96,22 \pm 8,94$ \\
\hline CA $(\mathrm{cm})$ & $92,45 \pm 15,80$ & $95,04 \pm 11,84$ & $100,72 \pm 9,51$ \\
\hline Variáveis hemodinâmicas & & & \\
\hline PAS $(\mathrm{mmHg})$ & $125,27 \pm 10,78$ & $114,25 \pm 14,02$ & $131,23 \pm 9,8$ \\
\hline PAD $(\mathrm{mmHg})$ & $70,62 \pm 8,61$ & $70,95 \pm 7,01$ & $76,39 \pm 4,15$ \\
\hline
\end{tabular}

G1=grupo1(Treinamento aeróbio); G2=grupo2(Treinamento resistido); G3=grupo3(Treinamento concorrente); $\mathrm{PAS}=$ pressão arterial sistólica; $\mathrm{PAD}=$ pressão arterial diastólica; $\mathrm{IMC}=$ índice de massa corporal; $\mathrm{RCQ}=$ relação cintura/quadril; $\mathrm{IC}=$ índice de conicidade; $\mathrm{CC}=$ circunferência da cintura; $\mathrm{CA}=$ circunferência do abdômen.

Tabela 2 - Comparação da pressão arterial e dos indicadores antropométricas no período pré e pós-treino em idosos normotensos submetidos a 36 sessões de treinamento aeróbio, resistido e concorrente.

\begin{tabular}{lccccccccc}
\hline \multicolumn{1}{c}{} & \multicolumn{7}{c}{ NORMOTENSOS } \\
\hline & G1 $(n=40)$ & & & G2 $(n=48)$ & & G3 $(n=45)$ \\
\hline PAS $(\mathrm{mmHg})$ & $119,1 \pm 6,57^{*}$ & $115,3 \pm 6,81$ & 0,000 & $103,1 \pm 5,77$ & $101,6 \pm 5,93$ & 0,104 & $123,3 \pm 9,10$ & $121,1 \pm 6,47$ & 0,228 \\
\hline PAD $(\mathrm{mmHg})$ & $70,2 \pm 6,96$ & $69,7 \pm 6,76$ & 0,385 & $66,3 \pm 4,91$ & $65,6 \pm 5,13$ & 0,176 & $75,7 \pm 4,94$ & $75,7 \pm 3,72$ & 0,971 \\
\hline IMC $\left(\mathrm{kg} / \mathrm{m}^{2}\right)$ & $25,97 \pm 5,23$ & $25,37 \pm 5,34$ & 0,218 & $25,66 \pm 4,39$ & $24,69 \pm 3,86$ & 0,067 & $33,26 \pm 3,47$ & $32,38 \pm 3,06$ & 0,199 \\
\hline RCQ & $0,83 \pm 0,11$ & $0,85 \pm 0,90$ & 0,729 & $0,9 \pm 0,23$ & $0,8 \pm 0,01$ & 0,466 & $0,87 \pm 0,04$ & $0,93 \pm 0,07$ & 0,082 \\
\hline IC & $1,16 \pm 0,19$ & $1,24 \pm 0,19$ & 0,253 & $1,22 \pm 0,2$ & $1,14 \pm 0,14$ & 0,338 & $1,52 \pm 01$ & $1,49 \pm 0,15$ & 0,719 \\
\hline CC $(\mathrm{cm})$ & $77,67 \pm 11,79$ & $83,17 \pm 11,6$ & 0,231 & $82,27 \pm 12,90$ & $76,87 \pm 8,44$ & 0,346 & $98,57 \pm 5,86$ & $97,57 \pm 9,13$ & 0,824 \\
\hline CA $(\mathrm{cm})$ & $86,92 \pm 17,18$ & $84,1 \pm 17,17$ & 0,215 & $87 \pm 9,76$ & $83,67 \pm 10,70$ & 0,210 & $105,52 \pm 4,32$ & $101,42 \pm 7,14$ & 0,074 \\
\hline
\end{tabular}

G1=grupo1(Treinamento aeróbio); G2=grupo2(Treinamento resistido); G3=grupo3(Treinamento concorrente); PAS= pressão arterial sistólica; $\mathrm{PAD}=$ pressão arterial diastólica; $I M C=$ índice de massa corporal; $R C Q=$ relação cintura/quadril; $I C=$ índice de conicidade; $C C=$ circunferência da cintura; $C A=$ circunferência do abdômen. * diferença significativa $p<0,05$.

Tabela 3 - Comparação da pressão arterial e dos indicadores antropométricos pré e pós-treino em idosos hipertensos submetidos a 36 sessões de treinamento aeróbio, resistido e concorrente.

\begin{tabular}{lccccccccc}
\hline & & \multicolumn{7}{c}{ HIPERTENSOS } \\
\hline & G1 $(n=47)$ & & & $G 2(n=45)$ & & $G 3(n=42)$ \\
\hline PAS $(\mathrm{mmHg})$ & $132,4 \pm 10,62^{*}$ & $124,9 \pm 9,1$ & 0,014 & $128,2 \pm 3,99^{*}$ & $126,3 \pm 4,15$ & 0,004 & $137,5 \pm 4,26 *$ & $135,2 \pm 3,54$ & 0,005 \\
\hline PAD $(\mathrm{mmHg})$ & $71 \pm 10,94$ & $69,9 \pm 9,27$ & 0,333 & $76,8 \pm 4,1$ & $76,6 \pm 3,22$ & 0,791 & $76,9 \pm 3,89$ & $77 \pm 4,65$ & 0,937 \\
\hline IMC $\left(\mathrm{kg} / \mathrm{m}^{2}\right)$ & $27,73 \pm 3,72$ & $25,64 \pm 7,89$ & 0,470 & $31,22 \pm 30,89$ & $30,89 \pm 4,02$ & 0,660 & $31,4 \pm 5,36$ & $31,60 \pm 5,38$ & 0,833 \\
\hline RCQ & $0,88 \pm 0,72$ & $0,90 \pm 0,1$ & 0,350 & $0,97 \pm 0,26$ & $0,96 \pm 0,05$ & 0,613 & $0,92 \pm 0,04$ & $0,9 \pm 0,46$ & 0,615 \\
\hline IC & $1,35 \pm 0,23$ & $1,39 \pm 0,27$ & 0,500 & $1,5 \pm 0,11$ & $1,53 \pm 0,14$ & 0,273 & $1,43 \pm 0,19$ & $1,4 \pm 0,2$ & 0,565 \\
\hline CC $(\mathrm{cm})$ & $89,97 \pm 14,14$ & $93,06 \pm 14,7$ & 0,249 & $98,16 \pm 6,03$ & $100,12 \pm 8,1$ & 0,280 & $94,34 \pm 11,14$ & $92,26 \pm 11,4$ & 0,524 \\
\hline CA $(\mathrm{cm})$ & $99,96 \pm 13,27$ & $97,65 \pm 11,8$ & 0,346 & $103 \pm 10,40$ & $103,4 \pm 10,1$ & 0,393 & $96,88 \pm 11,2$ & $80,96 \pm 42,1$ & 0,384 \\
\hline
\end{tabular}

G1=grupo1(Treinamento aeróbio); G2=grupo2(Treinamento resistido); G3=grupo3 (Treinamento concorrente); PAS= pressão arterial sistólica; $\mathrm{PAD}=$ pressão arterial diastólica; $I M C=$ índice de massa corporal; $R C Q=$ relação cintura/quadril; $I C=$ índice de conicidade; $C C=$ circunferência da cintura; $C A=$ circunferência do abdômen. * diferença significativa $p<0,05$. 


\section{DISCUSSÃO}

A prática de exercício físico como tratamento não farmacológico ou até mesmo sua realização em concomitância a utilização de medicamentos anti-hipertensivos, se encontra de forma sistemática na literatura como uma estratégia fundamental para a redução e manutenção da PA, seja de forma aguda ou crônica ${ }^{25,26}$.

No presente estudo que avaliou a resposta pressórica e adaptações morfológicas pós-exercício físico em normotensos e hipertensos medicamentados, submetidos a treinamento aeróbio, resistido e concorrente, foram observadas reduções da PA pós-exercício; entretanto, esta queda só pôde ser observada na PAS. Em contrapartida, no que diz respeito às adaptações morfológicas, estas mesmas evidências não foram visualizadas para nenhum dos grupos. No entanto, vale destacar os altos valores para as medidas do IMC e da $R C Q$, principalmente nos sujeitos hipertensos indo de encontro com alguns estudos que identificaram a associação da prevalência da gordura corporal com a hipertensão $0^{6,27}$.

$\mathrm{Na}$ análise da PA no G1, ficou evidenciado que os sujeitos normotensos apresentaram HPE significativa para o treinamento aeróbio, corroborando com o estudo de Tomasi et al. ${ }^{28}$, a investigar o comportamento da PA em sujeitos normotensos submetidos a exercícios aeróbios e resistidos, bem como houve HPE significativa para os hipertensos, indo de encontro com o estudo de Monteiro et al. ${ }^{13}$, com sujeitos hipertensos sob tratamento farmacológico, submetidos a 16 sessões de treinamento aeróbio. Este fato se apresenta em conformidade com alguns estudos na literatura que demonstram o efeito significativo do treinamento aeróbio para o controle da $\mathrm{PA}^{14,15,18}$. Este efeito hipotensor pode ser explicado em parte, devido ao recrutamento de grandes grupos musculares para realização do exercício aeróbio, no qual tendem a proporcionar melhores respostas hipotensoras $^{29}$, bem como o estimulo cardiorrespiratório, vascular e metabólico, já que o resultado hipotensor depende da redução do débito cardíaco, resultante em bradicardia de repouso ${ }^{12}$.

Apesar de muitos estudos analisarem a prática do exercício físico e sua relação com os níveis de PA pós-exercício utilizaram o exercício aeróbio como estratégia de intervenção, diversos são os estudos que buscam entender os efeitos hipotensores do exercício resistido ${ }^{1,30}$. Em estudos com sujeitos hipertensos submetidos a sessões de treino resistido ainda existe uma lacuna acerca do efeito hipotensor ${ }^{11}$, como nos achados de Costa et al. ${ }^{26}$, ao analisar o comportamento da PA após uma única sessão de treinamento resistido em idosas hipertensas, os quais obtiveram resultados significativos para a redução da $\mathrm{PA}$, corroborando com o presente estudo, enquanto que o estudo realizado por Canuto et al. ${ }^{30}$, os quais investigaram a influência do treinamento resistido sobre a PA de idosas hipertensas não houve resultados significativos para a PA pós-exercício.

O presente estudo traz um dado novo que ainda não tem grande repercussão na literatura, que é a redução da PA também em exercício concorrente, sendo observada uma diminuição da PAS em indivíduos hipertensos submetidos a este tipo de treinamento (G3); entretanto, vale ressaltar que os mesmos obtiveram estes resultados em quantidades de dias reduzidos, visto que os mesmos realizaram duas sessões diárias.

Como principais limitações do presente estudo pode-se destacar a aferição da PA pós-exercício realizada em um único momento, impossibilitando um acompanhamento da PA por um período mais prolongado, bem como a utilização e heterogeneidade dos medicamentos e a ausência de controle na dieta da amostra, 
impossibilitando maiores conclusões acerca do efeito hipotensor pós-exercício. Vale destacar como fator relevante deste estudo a utilização de duas sessões diárias de treinamento (aeróbio + resistido), fato este que não se apresenta difundido na literatura, possibilitando neste sentido novos estudos, a fim de se obter um maior esclarecimento do comportamento da PA para esta população submetida a este tipo de treinamento. Este estudo ainda se torna uma das poucas investigações na região Nordeste do Brasil a monitorar a PA em sujeitos submetidos a 36 sessões de treinamento, favorecendo para análises do efeito crônico do exercício físico no comportamento da PA.

Os dados deste estudo evidenciaram que a utilização de exercícios aeróbios, resistidos ou concorrentes resultaram em redução da PAS de forma significativa para os idosos hipertensos, enquanto que para os normotensos apenas o treinamento aeróbio apresentou queda na PAS. Entretanto, não se pôde observar decréscimo da PAD, bem como não houve efeito significativo sobre a morfologia dos idosos. Foi também possível destacar o efeito hipotensor em sujeitos hipertensos submetidos a treinamento concorrente, fato este não muito disseminado na literatura, possibilitando, desta forma, novos estudos com este tipo de treinamento, a fim de maiores esclarecimentos sobre o efeito deste na PA.

\section{REFERÊNCIAS}

1. Mediano MF, Paravidino V, Simão R, Pontes FL, Polito MD. Comportamento subagudo da pressão arterial após o treinamento de força em hipertensos controlados. Rev Bras Med Esporte 2005; 11: 337-40.

2. Cunha GA, Rios AC, Moreno JR, Braga PL, Campbelll CS, Simões HG, et al. Hipotensão pós-exercício em hipertensos submetidos ao exercício aeróbio de intensidades variadas e exercício de intensidade constante. Rev Bras Med Esporte 2006; 12: 313-7.

3. Kearney PM, Whelton M, Reynolds K, Muntner P, Whelton PK, He J. Global burden of hypertension: analysis of worldwide data. Lancet. 2005; 365: 217-23.

4. Zaitune MP, Barros MB, César CL, Carandina L, Goldbaum M. Hipertensão arterial em idosos: prevalência, fatores associados e práticas de controle no Município de Campinas, São Paulo, Brasil. Cad Saúde Pública 2006; 22:285-94.

5. Munaretti DB, Barbosa AR, Marucci MF, Lebrão ML. Hipertensão arterial referida e indicadores antropométricos de gordura em idosos. Rev Assoc Med Bras 2011; 57:25-30

6. Peixoto MR, Benício MH, Latorre MR, Jardim PC. Circunferência da cintura e índice de massa corporal como preditores da hipertensão arterial. Arq Bras Cardiol 2006; 87:462-70.

7. Borges HP, Cruz NC, Moura CE. Associação entre hipertensão arterial e excesso de peso em adultos, belém, pará, 2005. Arq Bras Cardiol 2008; 91:110-18.

8. Neter JE, Stam BE, Kok FJ, Grobbee DE, Geleijnse JM. Controlled trials influence of weight reduction on blood pressure: a meta-analysis of randomized. Hypertension. 2003; 42:878-84.

9. Benedetti TR, Meurer ST, Morini S. Índices antropométricos relacionados a doenças

10. Cardiovasculares e metabólicas em idosos. Rev. Educ. Fís/UEM 2012; 23:123-30.

11. Angad SS, Weltman A, Watson-Winfield D, Weltman J, Frick K, Patrie J, et al. Effect of fractionized vs continuous, singlesession exercise on blood pressure in adults J Hum Hypertens 2010; 24:300-2.

12. Polito MD, Simão R, Senna GW, Farinatti PT. Efeito hipotensivo do exercício de força realizado em intensidades diferentes e mesmo volume de trabalho. Rev Bras Med Esporte 2003; 9:69-73.

13. Rondom MU, Brum PC. Exercício físico como tratamento não-farmacológico da hipertensão arterial. Rev Bras Hipertens 2003; 10:134-39.

14. Monteiro HL, Rolim LM, Squinca DA, Silva FC, Ticianeli CC, Amaral SL. Efetividade de um programa de exercícios no condicionamento físico, perfil metabólico e pressão arterial de pacientes hipertensos. Rev Bras Med Esporte 2007; 13:107-12.

15. Casonatto J, Polito MD. Hipotensão Pós-exercício Aeróbio: Uma Revisão Sistemática. Rev Bras Med Esporte 2009; 15: 151-7. 
16. Cardoso CG, Gomides RS, Queiroz AC, Pinto LG, Lobo FS, Tinucci C, et al. Acute and chronic effects of aerobic and resistance exercise on ambulatory blood pressure. Clinics 2010; 65:317-25.

17. Gerage AM, Ciryno ES, Schiavoni D, Nakamura FY, Ronque ER, Gurjão AL, Gobbi S. Efeito de 16 semanas de treinamento com pesos sobre a pressão arterial em mulheres normotensas e não-treinadas. Rev Bras Med Esporte 2007; 13: 361-5.

18. Terra DF, Mota MR, Rabelo HT, Bezerra LM, Lima RM, Ribeiro AG, et al. Redução da pressão arterial e do duplo produto de repouso após treinamento resistido em idosas hipertensas. Arq Bras Cardiol 2008; 91:299-305.

19. Bermudes AM, Vassallo DV, Vasquez EC, Lima EG. Monitorização Ambulatorial da Pressão Arterial em Indivíduos Normotensos Submetidos a Duas Sessões Únicas de Exercícios: Resistido e Aeróbio. Arq Bras Cardiol 2003; 82:57-64.

20. kealey GA, Kealey KS. Progressive resistance exercise and resting blood pressure: a metaanalysis of randomized controlled trials. Hypertension 2000; 35:838-843.

21. Silva IC, Sartori M, Angelis K. Mecanismos hipotensores do exercício físico. Rev Bras Hipertens 2010; 13: 166-71.

22. ISAK. International standards for anthropometric assessment. Sydney: International Society for the Advancement of Kinanthropometry; 2001

23. Perloff D, Grim C, Flack J, Frohlich ED, Hill M, McDonald M, et al. Human blood pressure determination by sphygmomanometry. Circulation 1993; 88: 2460-70.

24. Baechle TR, Earle RW. Essentials of strength training and conditioning. 2 nd edition. Ed. Champaign IL: National Strength and Conditioning Association, 2000.

25. Santos EM, Simão R. Comportamento da pressão arterial após uma sessão de exercícios resistidos. Fit Perf J 2005; 4:227-31.

26. Rego AR, Gomes AL, Veras RP, Drummond E, Alkimin R, Dantas EH. Rev Bras Med Esporte 2011; 17:300-4.

27. Costa JB, Gerage AM, Gonçalves CG, Pina FL, Polito MD. Influência do Estado de Treinamento Sobre o Comportamento da Pressão Arterial Após uma Sessão de Exercícios com Pesos em Idosas Hipertensas. Rev Bras Med Esporte 2010; 16:103-6.

28. Rezende FA, Rosado LE, Ribeiro RC, Vidigal FC, Vasques AC, Bornad IS, et al. Índice de Massa Corporal e Circunferência Abdominal: Associação com Fatores de Risco Cardiovascular. Arq Bras Cardiol 2006; 87:728:34.

29. Tomasi T, Simão R, Polito MD. Comparação do comportamento da pressão arterial após sessões de exercício aeróbio e de força em indivíduos normotensos. Rev. Educ. Fís/UEM 2008; 19:361-7.

30. D’Assunção W, Daltro M, Simão R, Polito M, Monteiro W. Respostas cardiovasculares agudas no treinamento de força conduzido em exercícios para grandes e pequenos grupamentos musculares. Rev Bras Med Esporte 2007; 13:118-22.

31. Canuto PM, Nogueira ID, Cunha ES, Ferreira GM, Mendonça KM, Costa FA, et al. Influência do treinamento resistido realizado em intensidades diferentes e mesmo volume de trabalho sobre a pressão arterial de idosas hipertensas. Rev Bras Med 2011; 17:246-9.

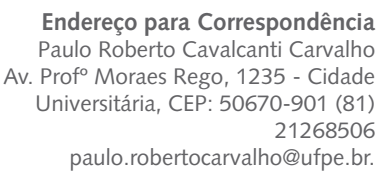

Recebido 13/08/2013

Revisado 31/08/2013

Aprovado 08/09/2013 